\title{
Differences in Volatile Profiles of Turnip Plants Subjected to Single and Dual Herbivory Above- and Belowground
}

\author{
Prisca S. Pierre • Jeroen J. Jansen • \\ Cornelis A. Hordijk • Nicole M. van Dam • \\ Anne-Marie Cortesero - Sébastien Dugravot
}

Received: 28 September 2010 /Revised: 31 January 2011 /Accepted: 8 March 2011 /Published online: 30 March 2011

(C) Springer Science+Business Media, LLC 2011

\begin{abstract}
Plants attacked by herbivorous insects emit volatile organic compounds that are used by natural enemies to locate their host or prey. The composition of the blend is often complex and specific. It may vary qualitatively and quantitatively according to plant and herbivore species, thus providing specific information for carnivorous arthropods. Most studies have focused on simple interactions that involve one species per trophic level, and typically have investigated the aboveground parts of plants. These investigations need to be extended to more complex networks that involve multiple herbivory above- and belowground. A previous study examined whether the presence of the leaf herbivore Pieris brassicae on turnip plants (Brassica rapa subsp. rapa) influences the response of Trybliographa rapae, a specialist parasitoid of the root feeder Delia radicum. It showed that
\end{abstract}

P. S. Pierre $(\varangle) \cdot$ A.-M. Cortesero $\cdot$ S. Dugravot

UMR 1099 BiO3P, University of Rennes 1, INRA,

Agrocampus Ouest,

263 avenue du Général Leclerc,

35042 Rennes Cedex, France

e-mail: pierreprisca@yahoo.fr

J. J. Jansen

Biosystems Data Analysis, Swammerdam Institute for Life

Siences (SILS), University of Amsterdam (UvA),

Nieuwe Achtergracht 166,

1018 WV Amsterdam, The Netherlands

C. A. Hordijk

Centre for Limnology,

Netherlands Institute of Ecology (NIOO-KNAW),

Rijksstraatweg 6,

3631AC Nieuwersluis, The Netherlands

N. M. van Dam

Institute for Water and Wetland Research (IWWR),

Radboud University Nijmegen,

PO Box 9010, 6500 GL Nijmegen, The Netherlands the parasitoid was not attracted by volatiles emitted by plants under simultaneous attack. Here, we analyzed differences in the herbivore induced plant volatile (HIPV) mixtures that emanate from such infested plants by using Orthogonal Partial Least Squares-Discriminant Analysis (OPLS-DA). This multivariate model focuses on the differences between odor blends, and highlights the relative importance of each compound in an HIPV blend. Dual infestation resulted in several HIPVs that were present in both isolated infestation types. However, HIPVs collected from simultaneously infested plants were not the simple combination of volatiles from isolated forms of above- and belowground herbivory. Only a few specific compounds characterized the odor blend of each type of damaged plant. Indeed, some compounds were specifically induced by root herbivory (4-methyltridecane and salicylaldehyde) or shoot herbivory (methylsalicylate), whereas hexylacetate, a green leaf volatile, was specifically induced after dual herbivory. It remains to be determined whether or not these minor quantitative variations, within the background of more commonly induced odors, are involved in the reduced attraction of the root feeder's parasitoid. The mechanisms involved in the specific modification of the odor blends emitted by dual infested turnip plants are discussed in the light of interferences between biosynthetic pathways linked to plant responses to shoot or root herbivory.

Key Words Plant-insect interactions · Multiple herbivory · Volatile organic compounds · Parasitoid - Trybliographa rapae $\cdot$ Delia radicum $\cdot$ Pieris brassicae $\cdot$ Brassicaceae

\section{Introduction}

Herbivory induces the emission of volatile organic compounds, which are called herbivore induced plant volatiles 
(HIPVs). Such chemicals are involved in plant indirect defense when they are used by the herbivores' natural enemies to locate their host or prey (e.g., Dicke and Sabelis, 1987; Geervliet et al., 1994; Turlings et al., 1995; Dicke and van Loon, 2000; Heil, 2008). The HIPV blends resulting from herbivore damage are not identical to those elicited by mechanical damage or released by intact plants (reviewed by Turlings et al., 1995; Takabayashi and Dicke, 1996). Indeed, mechanical wounding and chemical elicitors from insect oral secretions synergistically activate plant HIPV emissions (reviewed by Arimura et al., 2009). HIPVs are complex mixtures commonly consisting of 20 to over 200 compounds, ranging from simple alkanes to more elaborate structures such as sesquiterpenes (Dudareva et al., 2004). Such complexity may provide specific information to carnivorous arthropods and result in reliable cues for detection of suitable host or prey. The composition of the blend may vary according to plant and herbivore species, conditions, and developmental stage (Takabayashi et al., 1995; De Moraes et al., 1998; Gouinguené et al., 2003). Most published papers have described the role of HIPVs in simple tritrophic interactions that involve one species on each trophic level in the aboveground compartment of a plant system. However, roots can also emit HIPVs that attract enemies of belowground feeding herbivores (Aratchige et al., 2004; Rasmann et al., 2005; Ali et al., 2010). Therefore, more complex networks involving multiple herbivores should be studied (see Bezemer and van Dam, 2005; Dicke et al., 2009 for reviews). Feeding damage caused by root and shoot herbivores may lead to quantitative and qualitative changes of primary and secondary metabolites in both compartments of the plant (Bezemer and van Dam, 2005; van Dam et al., 2009). Such changes not only affect the development of herbivores in the two spatially separated compartments, but also the organisms on the third trophic level associated with these plants (Gols and Harvey, 2009). For instance, simultaneous feeding by aboveground herbivores (Spodoptera littoralis) and below-ground herbivores (Diabrotica virgifera virgifera $)$ in maize plants affects the production of a single HIPV, $(E)$ - $\beta$-caryophyllene, which in turn impacts on the behavior of respective natural enemies. This altered emission of volatiles reduced the attraction of the parasitic nematode that attacks the belowground herbivore (Rasmann and Turlings, 2007). Such changes in individual HIPV compounds may function synergistically with other less specific components as reliable cues for carnivores. For example, the homoterpene dimethyl-tridecatetraene (DMTT), is emitted by prey-infested lima bean plants (Phaseolus lunatus) but is not attractive to the predatory mite Phytoseilus persimilis as a pure compound. However, when it is added to a blend of compounds emitted from a plant infested by non-prey herbivores, predatory mites are attracted (Dicke et al., 1990). Thus, the specificity of each induced odor blend may be a function of the mixture, quantity or the relative amounts of the volatile compounds (De Boer et al., 2004; Mumm and Hilker, 2005). Plant volatile emissions should always be considered in the context of all the other volatiles of the blend (van Dam and Poppy, 2008; van Dam et al., 2010; Ranganathan and Borges, 2010). HIPV emissions thus require multivariate analysis to allow understanding in terms of insect behavior. Furthermore, herbivores not only increase the emission of certain compounds but also can reduce the release of some wound-elicited constituents (Gaquerel et al., 2009). Although there are few examples in the literature, blends of HIPVs also may differ qualitatively and then provide specificity of chemical cues in host location by parasitoids (Takabayashi et al., 2006). Both quantity and quality of the volatiles perceived by natural enemies may determine the reliability of the informative odor (Vet et al., 1991).

Delia radicum, the cabbage root fly, is a major pest of brassicaceous crops. Females aggregatively lay their eggs near plant stems, and the larvae crawl down to feed on the roots and develop until they pupate in the soil. These larvae frequently are parasitized by Trybliographa rapae, a specific larval endoparasitoid of Delia species. This parasitoid forages aboveground for hosts that are feeding on plant roots belowground; therefore, systemically emitted volatiles play a key role in its host location. Females are attracted by volatiles emitted by undamaged leaves of $D$. radicum infested plants but not by volatiles emitted by leaves of artificially damaged plants (Neveu et al., 2002). Furthermore, volatiles emitted by leaves of plants artificially damaged at the roots to which crushed larvae are applied are attractive; this suggests that the attractive blend of HIPVs produced is specific to $D$. radicum feeding activity (Neveu et al., 2002). The specific composition of the HIPV blend elicited by $D$. radicum feeding on turnips and involved in $T$. rapae attraction has not been determined. A recent study showed that when Pieris brassicae (a specialist leaf feeder of brassicaceous plants) and $D$. radicum simultaneously attack a turnip plant (Brassica rapa subsp. rapa), T. rapae is no longer attracted (Pierre et al., 2011). Reduced attraction of root and shoot infested plants in olfactometer experiments was correlated with lower levels of parasitism in a separate field study (Pierre et al., 2011).

In this study, we analyzed the blends of HIPVs emitted by turnip plants after (1) infestation by $D$. radicum, (2) infestation by $P$. brassicae, (3) simultaneous infestation by $D$. radicum and $P$. brassicae, or (4) no infestation. The combination of experiments revealed specific modifications of HIPV blends following single and dual infestations. This is a first step towards understanding the altered foraging behavior of T. rapae in the presence of leaf herbivory. The analysis of HIPVs needs a multivariate approach in which 
the relative amounts of volatiles are as important as the amounts themselves. Therefore, Orthogonal Partial Least Squares-Discriminant Analysis (OPLS-DA) was used to observe the effect of different forms of herbivore infestation on HIPV emission blends. The resulting model gives the relative importance of each volatile in a system-wide model describing the entire blend. It optimally describes the effect of each infestation as a classification between control and treated plants. However, OPLS-DA does not assess differences in the patterns of HIPV emissions between single herbivore infestations and dual herbivory. So it does not explicitly reveal the synergistic or antagonistic effects of additional herbivory. To address this question, univariate analysis was used to evaluate a posteriori the interaction between leaf and root herbivory effects on the levels of important HIPVs.

\section{Method and Materials}

Plants Cultivated turnip plants, Brassica rapa subsp. rapa (var. Nancy) were used. Seeds were sown in compost-soil, and after 10 days, seedlings were transplanted into $9 \times 9 \times$ $9 \mathrm{~cm}$ plastic pots, filled with sandy soil. Plants were grown in a greenhouse, at $60 \%$ r.h., $21^{\circ} \mathrm{C} / 16^{\circ} \mathrm{C}, 16 / 8 \mathrm{~h} \mathrm{~L}: \mathrm{D}$ photoperiod. Natural day-light was supplemented when needed with metal halide lamps $\left(200 \mu \mathrm{mol} / \mathrm{s} / \mathrm{m}^{2}\right.$ PAR $=$ photosynthetically active radiation; 1 lamp per $\mathrm{m}^{2}$ ). Plants were watered every day and supplemented with nutrients (4 $\mathrm{N}: 6 \mathrm{P}: 8 \mathrm{~K}$ and micronutrients) twice a week until they were 7- to 8-wk-old.

Insects Delia radicum larvae used for plant infestation originated from our rearing. The populations were originally established in the laboratory from flies collected in the field at St Méloir des Ondes (Brittany, France) during the summer of 1994. The brood was reared as described in Neveu et al. (1996) and supplemented yearly with new field-captured individuals coming from the same region. Pieris brassicae caterpillars originated from an insect culture maintained at the Laboratory of Entomology of Wageningen University, the Netherlands, and were reared on Brassica oleracea subsp. gemmifera (Brussel sprout) plants.

Induction Procedure Plants were infested when they had five to eight fully expanded leaves (7-8-wk old). Twelve to thirteen plants of similar size and stature were selected for each of the different treatment groups. Infestation with $P$. brassicae $(\mathrm{P})$ consisted of placing ten first instars with a small brush on the two youngest leaves ( 5 per leaf) of each plant. Plants were infested by $D$. radicum (D) by placing $5 \mathrm{~s}$ instars with a brush onto the soil surface, immediately adjacent to the stem of each plant. Plants were checked $1 \mathrm{~h}$ later to control whether all larvae had disappeared into the sand. With dual infestation (DP), larvae of $P$. brassicae (see above) were applied first to each plant followed $1 \mathrm{~h}$ later by D. radicum larvae. A nutrient solution $(4 \mathrm{~N}: 6 \mathrm{P}: 8 \mathrm{~K}$ and micronutrients) then was applied to both infested and uninfested plants.

Collection of Volatiles and GC-MS Analysis Volatiles were collected 3 days after induction by infestation. Based on our previous studies with turnip plants, 3 days of infestation sufficed for effective induction of $T$. rapae attracting volatiles (Neveu et al., 2002; Pierre et al., 2011). The entire plants (including pots and sand) were transferred to four separate 171 glass bell-shaped collection chambers placed in a controlled climate cabinet $\left(21^{\circ} \mathrm{C}, 70 \%\right.$ r.h. $)$. The chambers were supplied constantly at the top with pressurized air (Hoek Loos, NL) cleaned over a Zero Air generator to remove hydrocarbons (Parker Hannifin Corp, Tewksbury, MA, USA). Plant-generated HIPVs were collected over a steel trap filled with $150 \mathrm{mg}$ Tenax TA and $150 \mathrm{mg}$ Carbopack B by using a vacuum pump. Collection flow rates were set to $100 \mathrm{ml} / \mathrm{min}$. The HIPVs were collected from an outlet on the side of the flask, which was at the same height as the plant leaves. The supply rate of clean air at the top of the flask was set to $200 \mathrm{ml} / \mathrm{min}$. After $1 \mathrm{~h}$, the traps were removed from the pump and capped until analysis. In this way, within 1 days we measured six full series of four plants representing the four different treatments tested and two background HIPV profiles from an empty glass chamber. We sampled the following numbers of individual plants: 13D, 13P, 12DP, and $13 \mathrm{C}$. Volatiles were desorbed from the traps, then analyzed by GC-MS using the same methods and reference compounds as described by van Dam et al. (2010). All integrated signals were generated from the MSchromatograms by the AMDIS software, except those of alpha-pinene, isobutyl isothiocyanate, and 2-isothiacyanatobutane, which were extracted as ion traces from the same peak from which they co-eluted by Xcalibur software. Note that these three compounds co-eluted so close that they could not be separated even by deconvolution. However, strong unique ions were found in the mass spectrum of the mixture (alpha-pinene, 93; isobutyl isothiocyanate, 115, 2isothiacyanato-butane, 86) and relative trends generated from these single qualifier ions are in theory proportional to trends in the total ion traces.

Peak areas obtained in each sample were divided by the total volume in $\mathrm{ml}$ that was sampled in the trap, to correct for minor differences in sampling time and flow rates over individual traps.

Data Analysis of Volatile Profiles Peaks relating to mixtures and impurities were removed from the dataset. 
Furthermore, to avoid rare compounds that disproportionably could affect the outcome, only those compounds that were found at least six times in at least one treatment group were retained for the multivariate analyses. This resulted in a dataset of 37 HIPVs for detailed analysis. We constructed three separate models, each of which compared the HIPV profiles of one form of herbivore infestation to the control plants, thereby isolating the effect of one herbivory form on the HIPV composition. For these models, the Orthogonal Partial Least Squares-Discriminant Analysis (OPLS-DA) method was chosen. It takes existing biochemical relations among the volatile compounds into account, and the interpretation of results from the model compare well in terms of the differences among the plant groups (Bylesjö et al., 2006). In brief, OPLS-DA is a supervised component analysis method that uses a priori knowledge about the form of herbivory that every plant received. The models consist of a small number of 'Latent Variables' - comparable to the components of Principal Component Analysiswhich together describe the difference between control and treated plants. The models in this study consisted of a description of the treatment each plant most likely received (the class membership description, $\gamma_{\text {hat }}$ ) and the metabolic profile with relative importance of each volatile in the total blend (the OPLS-DA weight vector). Although OPLS-DA models may contain multiple latent variables, there is always only one class prediction and one weight vector per plant when two groups are compared.

The OPLS-DA model thereby specifically focuses on differences among plant groups, and like all supervised models it is thereby prone to 'over fit' these differences, i. e., describing the variation among the individual plants used in the experiment, rather than generic phenomena caused by the experimental manipulations (Jansen et al., 2010). Therefore, the observations from the resulting models were evaluated thoroughly by cross-validation, in which the data of randomly chosen individual plants are left out of the data set and the model is re-made, after which the treatment of the left-out plants is estimated. This procedure was repeated 2,000 times, which then leads to a misclassification rate; a measure of model error. The number of latent variables for which the misclassification rate is lowest results in a model that includes all systematic information useful for predicting treatment groups, but does not contain superfluous 'random' information that relates to over fit. As a second validation, the determined misclassification rate then was compared to that of a large number of models in which treatments were artificially shuffled by permutation: this naturally should lead to considerably higher misclassification, because true biological difference between the permuted treatments is lacking. The permutation is repeated 1,000 times, each time consisting of 2,000 cross-validated models providing a misclassification rate. The difference in misclassification rate between the model with real and permuted treatments can be expressed as a $P$-value that describes the likelihood that the observed effect is real.

The chemistry in the 'weight' vector of treatments with a significant effect on HIPV emissions was analyzed further. This vector-showing the importance of each volatile in describing the differences between two groups- was compared to those obtained from the models on permutated treatments described before. These permutated models collectively form a 'null distribution' of the OPLS-DA weights for every volatile, from which the weight of the model fitted on real treatments should differ. This last permutation analyses results in a $P$-value for every volatile for each form of herbivory.

In our case, there were three separate OPLS-DA models that we compared for communalities and differences caused by different forms of herbivory. This comparison was done by plotting the weight vectors of each volatile on the first latent variable (LV) for each treatment. However, threedimensional figures that compare the different forms of herbivory are difficult to interpret. By condensing the relevant information to a two-dimensional figure, it becomes more insightful. This figure, called a bubble plot (Markus and $\mathrm{Gu}, 2010$ ), compares the chemistry underlying two forms of herbivory as positions of each volatile on two axes (i.e., a scatter plot). In addition, the amount of volatile from the third form of herbivory is represented as the area of a circle: this graphical tool is useful for comparing both forms of individual herbivory and the third, combined infestation.

Afterwards, compounds of which the weight vector values were significant for at least one form of herbivory were selected for univariate analysis. Indeed, for each important compound, we tested the effect of root herbivory (D), leaf herbivory $(\mathrm{P})$, and the interaction $\left(\mathrm{D}^{*} \mathrm{P}\right)$ by using two-way analysis of variance on peak areas transformed as $\log (\mathrm{x}+1)$ (van Dam et al., 2010). The significance of such an interaction is of interest, as it could reveal a positive or negative influence of dual herbivore treatment (DP) compared to a single herbivore one ( $\mathrm{P}$ or $\mathrm{D})$. A similar procedure was used previously by Soler et al. (2007a) to support the results of their multivariate analysis. In the present study, homogeneity of variance was checked by Levene's test using the "robvar" function in Stata 9 software (StataCorp LP, College Station, TX, USA).

\section{Results}

The fitted OPLS-DA models showed that the effect of all three herbivore treatments on the HIPV emissions can be described 
by simple low-dimensional multivariate models. The crossvalidation yielded a single latent variable for all three to significantly distinguish the volatile blends emitted by turnip plants (Table in Fig. 1). The comparison of these three separate OPLS-DA models for communalities and differences caused by different forms and levels of herbivory was done by plotting the discriminate functions of root herbivory by $D$. radicum (D) and leaf herbivory by $P$. brassicae $(\mathrm{P})$ against each other (Fig. 1). Increased levels of a compound in $\mathrm{D}$ or $\mathrm{P}$ are indicated by a positive weight vector value on the respective axis, while reduced levels are shown by a negative value. The size of each bubble corresponds to the weight vector value of a volatile for the dual herbivory treatment (DP). The maximal bubble size corresponds to (3E)-4,8-dimethyl-1,3,7-nonatriene DMNT (2), which then is related to the highest vector value for DP (0.394) and to the most significant difference from the vector value for the control $(P=0.004)$. The absolute minimal bubble size is associated with heptadecene (29), which has the lowest absolute vector value for DP (0.002) and the least significant difference from the vector value for the control $(P=0.595)$. The bubble size of hexylacetate (9) represents a threshold of significance (weight vector value $=0.300 ; P=0.044$ ) of weight vector values associated with DP.

For instance, the level of $(E, E)$-4,8,12-trimethyl1,3,7,11-tridecatetraene TMTT (1) increased after any form of herbivory, as indicated by its high and positive weight vector value on the $\mathrm{D}$ axis $(0.276)$, the $\mathrm{P}$ axis (0.329) and the large black bubble indicating a high and positive weight vector value for (DP) (0.305). By contrast, the level of 2undecanone (20) was mildly increased by (D), which is represented by a relatively high and positive value in the $\mathrm{D}$ axis (0.196), but this did not respond for (P) and (DP) as indicated by a low weight vector in $\mathrm{P}$ axis $(-0.004)$ and a small size of the bubble meaning a low weight vector value for (DP) (-0.055). An example of a compound whose level mildly decreased in (P) is 1-hexadecanol (12) which has a negative value on the $\mathrm{P}$ axis $(-0.083)$; this compound did not respond to (D) or (DP), as revealed by the low value in the $\mathrm{D}$ axis $(0.008)$ and the small size of the bubble (0.005) (Fig. 1).

Hence, the bubble plot representation allows visual identification of interesting compounds that are important for differentiating between D, P, and DP volatile profiles. In particular, the right upper quarter of the bubble plot shows the compounds, TMTT (1), DMNT (2), (E,E)-alphafarnesene (6), hexylacetate (9), and (E)-3-hexen-ol-acetate (10) the levels of which were relatively high in all three herbivore treatments as indicated by the large filled dots (Fig. 1). Interestingly, in the same quarter of the figure, smaller black dots indicate compounds that increased in $\mathrm{P}$ or D but not in DP, i.e., methysalicylate (7) and 4methyltridecane (28) (Fig. 1). Consequently, the latter
DP Axis (1LV, $P=0.001)$

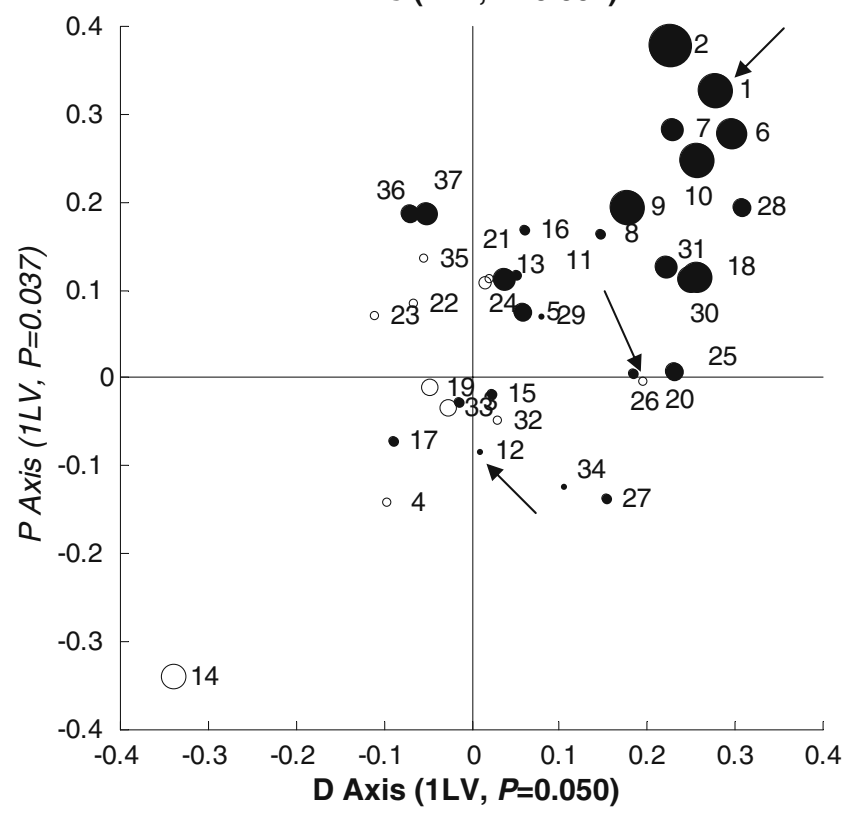

\begin{tabular}{|c|c|c|}
\hline treatment & number of LVs & $\boldsymbol{P}$-values \\
\hline (D) & 1 & 0.050 \\
\hline (P) & 1 & 0.037 \\
\hline (DP) & 1 & 0.001 \\
\hline
\end{tabular}

Fig. 1 Two dimensional bubble plot representation of volatiles emitted after different forms of herbivore infestations in Brassica rapa subsp. rapa plants: discriminate functions of root herbivory by Delia radicum larvae (D) and leaf herbivory by Pieris brassicae caterpillars $(\mathrm{P})$ plotted against each other. Numbers correspond to compounds listed in Table 1. The size of each bubble representing a volatile corresponds to the weight vector value of that volatile for the dual herbivory treatment, i.e., herbivory by Delia radicum larvae and Pieris brassicae caterpillars (DP). The color shows if the volatile increases (filled) or decreases (open) in the DP treatment. The three arrows indicate examples of compounds given in the text. The table below presents the number of latent variables (LVs) prescribed by cross-validation and the $P$-values for the significance $(P \leq 0.050)$ of the system-wide effect brought about by each form of herbivory

compounds are compounds that would differentiate between single herbivory and dual herbivory effects on the plant volatile profile.

Volatile Profiles of Plants Exposed to Root Herbivory (D) Permutation tests of weight vector values showed that the level of 4-methyltridecane (28) was higher in plants exposed to herbivory by $\mathrm{D}$ alone compared to control plants (Table 1). This compound appeared to be specific for the volatile blend emitted by (D), as its level was significantly modified in D plants but neither in P nor DP plants. The levels of the homoterpene TMTT (1) and the sesquiterpene (E,E)-alpha-farnesene (6) also were higher, 
whereas that of the alcohol 2-octen-1-ol (14) was lower in plants exposed to (D) (Table 1). In addition to these significant differences, a marginal increase (arbitrary threshold for marginal variations: $P \leq 0.065)$ was observed for salicylaldehyde (18) and the green leaf volatile $(E)-3$ hexen-1-ol-acetate (10) (Table 1). All those compounds appeared to be important in characterizing the volatile blend emitted by root infested plants, since their corresponding weight vectors had either high or low values compared to control plants (Fig. 1).

Volatile Profile of Plants Exposed to Leaf Herbivory $(P)$ The level of methylsalycilate (7) significantly increased after (P) compared to control plants (Table 1). This phenomenon is specific for the odor blend emitted by leaf infested plants, as it does not appear in the other forms of herbivore treatment. Similar to (D), the volatile emissions of TMTT (1) and (E,E)-alpha-farnesene (6) significantly increased, whereas they decreased for 2-octen-1-ol (14) in plants exposed to (P) (Table 1). In addition, the levels of DMNT (2), and of the green leaf volatile (E)-3-hexen-1-ol acetate (ten marginally, i.e., $P \leq 0.065$ ) increased after $(\mathrm{P})$ compared to control plants (Table 1).

Volatile Profile of Plants Exposed to Both Forms of Herbivory (DP) The level of hexylacetate (9) was significantly higher in (DP) compared to control plants (Table 1). This is specific for the volatile blend from dual infested plants. Indeed, no significant increase in hexylacetate was observed in (P) or (D), (Table 1). Furthermore, some compound levels in the headspace of DP followed patterns similar to single infested plants, either with D or P. This was true for increased levels of TMTT (1), (E)-3-hexen-1ol-acetate $(10)$ and, marginally so (i.e., $P \leq 0.065)$ for $(E, E)$ alpha-farnesene (6) (Table 1). DMNT (2) also increased in DP plants, which followed the same tendency as $\mathrm{P}$ (Table 1). By contrast, lower levels of 2-octen-1-ol (14) were observed both in (D) and (P), but not in (DP), despite a similar tendency in the single infestations (Table 1).

In summary, both the bubble plot and permutation tests show that the plant response to dual herbivory (DP) is different from the combined effects of $D$. radicum and $P$. brassicae feeding alone (Fig. 1), despite a few communal patterns.

Effect of Interactions Between Root (D) and Leaf Herbivory $(P)$ Only compounds of which the weight vector values were found to be significant for at least one form of herbivory were selected for univariate analysis to assess the interaction between (D) and (P) effect (Table 2). ANOVA analysis (Table 2) showed a significant interaction between (D) and (P) effects on 4-methyltridecane (28). This implies that the positive effect of (D) on the emission of this compound was reduced in the presence of $(\mathrm{P})$ (Table 1, Table 2). Together with the bubble plot (mentioned above), such interaction indicates that the effect of root herbivory on this compound was different from the effect of dual herbivory. This confirms the high specificity of this compound for turnip plants infested by the root herbivore alone. In addition, an antagonistic effect between $\mathrm{D}$ and $\mathrm{P}$ was observed for 2-octen-1-ol (14; Table 1, Table 2). Indeed, the concentration of this compound was reduced in plants infested by D or P alone (as already mentioned) but not in dual infested plants.

The positive effect of $\mathrm{P}$ on the levels of methylsalycilate (7; Table 1) was significantly reduced by the additional presence of D. In other words, the effect of $\mathrm{P}$ was different from the effect of DP, as shown by the bubble plot (Fig. 1 mentioned above) and the significant interaction between $\mathrm{D}$ and $\mathrm{P}$ (Table 2). This confirms the specificity of methylsalycilate (7) induction as a cue for the leaf herbivores on plants without root damage. In addition, a significant interaction between $\mathrm{D}$ and $\mathrm{P}$ was observed for $(E, E)$-alpha-farnesene (6) (Tables 1,2$)$. The interaction was suggested by a marginal (i. e., $P \leq 0.065)$ although not significantly higher level of $(E, E)$ alpha-farnesene in dual infested plants compared to control plants. Permutation tests revealed higher levels of hexylacetate (9) in DP compared to control plants (Table 1), which is specific for such herbivore treatment since no significant increases were observed in $\mathrm{D}$ and $\mathrm{P}$ plants. However, ANOVA did not show any significant effects of interaction between $\mathrm{D}$ and $\mathrm{P}$ on that compound.

\section{Discussion}

Our results show that higher levels of the branched alkane, 4methyltridecane were highly specific for the volatile blend of plants infested by the root herbivore alone. Different alkanes, including branched alkanes, already have been detected in leaf and floral headspace of brassicaceous plants (Jakobsen et al., 1994; Gruber et al., 2009). However, we are unaware of any publications on the specific involvement of alkanes in the attraction of natural enemies of herbivores. In addition, salicylaldehyde emissions were marginally $(P=0.058)$ modified by root herbivore feeding. Salicylaldehyde already has been reported to play an important role in some tritrophic systems that involve leaf beetle larvae (Chrysomela spp.) and their natural enemies (reviewed by Gross and Schmidtberg, 2009). The two specific HIPVs, 4-methyltridecane and salicylaldedhyde, thus, could serve as cues for the parasitoid $T$. rapae when searching for $D$. radicum infested plants without leaf herbivores present as well. Further investigations based for example on gas chromatography coupled to electroantennography (GC-EAG) could reveal which compounds 
Table 1 Peak areas transformed as $\log (\mathrm{X}+1)$ of volatile compounds from Brassica rapa subsp. rapa plants exposed to root herbivory by Delia radicum (D) or leaf herbivory by Pieris brassicae (P), dual herbivory by Delia radicum and Pieris brassicae (DP), or no herbivory (control)

\begin{tabular}{|c|c|c|c|c|c|c|c|c|c|c|}
\hline No. & Compound name ${ }^{\mathrm{a}}$ & $\mathrm{RI}^{\mathrm{b}}$ & $\begin{array}{l}\text { Compound } \\
\text { class }\end{array}$ & Control & $\mathrm{D}$ & $\mathrm{P}$ & $\mathrm{DP}$ & $\begin{array}{l}P \\
(\mathrm{D})^{\mathrm{c}}\end{array}$ & $P(\mathrm{P})$ & $\begin{array}{l}P \\
\text { (DP) }\end{array}$ \\
\hline 1 & $\begin{array}{l}(E, E)-4,8,12 \text {-Trimethyl- } \\
\text { 1,3,7,11-tridecatetraene } \\
\text { (TMTT) }\end{array}$ & 1577 & Homoterpene & $0.00 \pm 0.00$ & $0.87 \pm 0.38$ & $1.52 \pm 0.41$ & $1.17 \pm 0.42$ & 0.046 & 0.011 & 0.022 \\
\hline 2 & $\begin{array}{c}\text { (3E)-4,8-Dimethyl-1,3,7- } \\
\text { nonatriene (DMNT) }\end{array}$ & 1117 & Homoterpene & $0.92 \pm 0.40$ & $2.06 \pm 0.48$ & $3.32 \pm 0.32$ & $3.10 \pm 0.30$ & 0.108 & 0.004 & 0.004 \\
\hline 3 & alpha-Pinene* & 932 & Monoterpene & $1.89 \pm 0.08$ & $1.88 \pm 0.04$ & $1.86 \pm 0.07$ & $1.91 \pm 0.06$ & 0.483 & 0.448 & 0.427 \\
\hline 4 & Limonene* & 1026 & Monoterpene & $3.33 \pm 0.09$ & $3.25 \pm 0.05$ & $2.97 \pm 0.26$ & $3.26 \pm 0.07$ & 0.311 & 0.268 & 0.319 \\
\hline 5 & Caryophyllene & 1413 & Sesquiterpene & $0.83 \pm 0.44$ & $1.11 \pm 0.48$ & $1.27 \pm 0.46$ & $1.85 \pm 0.57$ & 0.443 & 0.339 & 0.151 \\
\hline 6 & $(E, E)$-alpha-Farnesene* & 1506 & Sesquiterpene & $2.11 \pm 0.41$ & $3.43 \pm 0.33$ & $3.69 \pm 0.35$ & $3.39 \pm 0.36$ & 0.023 & 0.044 & 0.055 \\
\hline 7 & Methylsalicylate & 1191 & Ester & $1.00 \pm 0.37$ & $1.97 \pm 0.39$ & $2.45 \pm 0.32$ & $1.87 \pm 0.41$ & 0.111 & 0.046 & 0.145 \\
\hline 8 & Ethylacetate & 612 & Ester & $1.94 \pm 0.61$ & $2.87 \pm 0.55$ & $3.16 \pm 0.50$ & $2.09 \pm 0.63$ & 0.226 & 0.126 & 0.492 \\
\hline 9 & Hexylactetate & 1015 & Ester & $2.29 \pm 0.37$ & $2.92 \pm 0.27$ & $3.17 \pm 0.29$ & $3.42 \pm 0.10$ & 0.178 & 0.113 & 0.044 \\
\hline 10 & (Z)-3-Hexen-1-ol-acetate & 1008 & Ester & $4.36 \pm 0.13$ & $4.71 \pm 0.10$ & $4.82 \pm 0.13$ & $4.88 \pm 0.11$ & 0.054 & 0.061 & 0.016 \\
\hline 11 & 1-Tetradecanol & 1675 & Alcohol & $1.47 \pm 0.46$ & $1.72 \pm 0.46$ & $2.13 \pm 0.42$ & $1.76 \pm 0.45$ & 0.425 & 0.237 & 0.417 \\
\hline 12 & 1-Hexadecanol & 1881 & Alcohol & $2.91 \pm 0.26$ & $2.94 \pm 0.38$ & $2.53 \pm 0.43$ & $2.93 \pm 0.28$ & 0.465 & 0.301 & 0.488 \\
\hline 13 & 3-Hexen-1-ol & 850 & Alcohol & $2.99 \pm 0.37$ & $3.07 \pm 0.39$ & $3.49 \pm 0.30$ & $2.54 \pm 0.54$ & 0.404 & 0.237 & 0.251 \\
\hline 14 & 2-Octen-1-ol & 1068 & Alcohol & $2.08 \pm 0.34$ & $0.64 \pm 0.34$ & $0.40 \pm 0.27$ & $0.92 \pm 0.40$ & 0.014 & 0.008 & 0.075 \\
\hline 15 & 1-Hexanol & 865 & Alcohol & $2.50 \pm 0.21$ & $2.55 \pm 0.22$ & $2.44 \pm 0.31$ & $2.63 \pm 0.24$ & 0.359 & 0.500 & 0.311 \\
\hline 16 & 1-Octanol & 1071 & Alcohol & $2.71 \pm 0.34$ & $2.90 \pm 0.25$ & $3.25 \pm 0.05$ & $2.94 \pm 0.27$ & 0.468 & 0.230 & 0.453 \\
\hline 17 & 1-Dodecanol & 1474 & Alcohol & $3.06 \pm 0.14$ & $2.84 \pm 0.28$ & $2.86 \pm 0.27$ & $3.08 \pm 0.11$ & 0.311 & 0.371 & 0.473 \\
\hline 18 & Salicylaldehyde & 1038 & Aldehyde & $0.49 \pm 0.26$ & $1.33 \pm 0.31$ & $0.91 \pm 0.30$ & $1.38 \pm 0.30$ & 0.058 & 0.233 & 0.080 \\
\hline 19 & Octadecanal & 2021 & Aldehyde & $1.95 \pm 0.45$ & $1.72 \pm 0.47$ & $1.89 \pm 0.44$ & $1.03 \pm 0.44$ & 0.455 & 0.486 & 0.161 \\
\hline 20 & 2-Undecanone & 1293 & Ketone & $0.91 \pm 0.40$ & $1.80 \pm 0.41$ & $0.89 \pm 0.39$ & $0.67 \pm 0.35$ & 0.122 & 0.483 & 0.376 \\
\hline 21 & 2,4-Pentadione & 789 & Ketone & $2.47 \pm 0.48$ & $2.67 \pm 0.52$ & $3.12 \pm 0.40$ & $3.33 \pm 0.31$ & 0.386 & 0.280 & 0.185 \\
\hline 22 & $(E)$-Geranyl acetone & 1451 & Ketone & $3.74 \pm 0.05$ & $3.71 \pm 0.06$ & $3.81 \pm 0.07$ & $3.70 \pm 0.06$ & 0.374 & 0.318 & 0.323 \\
\hline 23 & 2-Nonanone & 1091 & Ketone & $2.28 \pm 0.29$ & $1.88 \pm 0.37$ & $2.51 \pm 0.22$ & $2.17 \pm 0.30$ & 0.303 & 0.379 & 0.406 \\
\hline 24 & Acetone & 516 & Ketone & $3.78 \pm 0.60$ & $3.88 \pm 0.61$ & $4.48 \pm 0.38$ & $2.88 \pm 0.74$ & 0.400 & 0.309 & 0.269 \\
\hline 25 & 6-Methyldodecane & $1253 \mathrm{a}$ & Alkane & $1.60 \pm 0.43$ & $2.61 \pm 0.33$ & $1.64 \pm 0.44$ & $2.32 \pm 0.41$ & 0.092 & 0.424 & 0.204 \\
\hline 26 & 3-Methylundecane & $1176 \mathrm{a}$ & Alkane & $1.77 \pm 0.41$ & $2.51 \pm 0.31$ & $1.8 \pm 0.41$ & $1.95 \pm 0.42$ & 0.167 & 0.455 & 0.421 \\
\hline 27 & 2,6-Dimethylundecane & $1212 \mathrm{a}$ & Alkane & $1.56 \pm 0.49$ & $2.36 \pm 0.46$ & $0.76 \pm 0.40$ & $1.99 \pm 0.51$ & 0.209 & 0.215 & 0.382 \\
\hline 28 & 4-Methyltridecane & $1358 \mathrm{a}$ & Alkane & $1.47 \pm 0.40$ & $2.68 \pm 0.23$ & $2.41 \pm 0.30$ & $2.18 \pm 0.38$ & 0.026 & 0.089 & 0.194 \\
\hline 29 & Heptadecene & 1692 & Alkene & $1.33 \pm 0.49$ & $1.74 \pm 0.47$ & $1.77 \pm 0.48$ & $1.34 \pm 0.48$ & 0.312 & 0.338 & 0.595 \\
\hline 30 & 2,4-Dithiapentane & 882 & Sulfide & $0.48 \pm 0.33$ & $1.58 \pm 0.43$ & $1.06 \pm 0.46$ & $1.53 \pm 0.48$ & 0.074 & 0.301 & 0.111 \\
\hline 31 & Dimethyldisulfide* & 738 & Sulfide & $3.08 \pm 0.28$ & $3.62 \pm 0.11$ & $3.43 \pm 0.11$ & $3.60 \pm 0.09$ & 0.101 & 0.289 & 0.138 \\
\hline 32 & Hexanoic acid & 981 & Acid & $1.50 \pm 0.41$ & $1.63 \pm 0.44$ & $1.26 \pm 0.39$ & $1.13 \pm 0.41$ & 0.414 & 0.415 & 0.334 \\
\hline 33 & Acetic acid & 568 & Acid & $3.50 \pm 0.44$ & $3.36 \pm 0.53$ & $3.30 \pm 0.53$ & $2.72 \pm 0.59$ & 0.473 & 0.48 & 0.245 \\
\hline 34 & Indole* & 1287 & Indole & $1.31 \pm 0.35$ & $1.69 \pm 0.33$ & $0.77 \pm 0.33$ & $1.34 \pm 0.35$ & 0.299 & 0.203 & 0.508 \\
\hline 35 & $\begin{array}{l}\text { Dihydroxybenzoquinone } \\
\text { (DBQ) }\end{array}$ & 1462 & Unknown & $1.80 \pm 0.42$ & $1.56 \pm 0.42$ & $2.47 \pm 0.32$ & $1.49 \pm 0.45$ & 0.408 & 0.193 & 0.359 \\
\hline 36 & Isobutyl isothiocyanate & 931 & Isothiocyanate & $1.06 \pm 0.15$ & $0.94 \pm 0.16$ & $1.47 \pm 0.17$ & $1.27 \pm 0.10$ & 0.341 & 0.114 & 0.201 \\
\hline 37 & 2-Isothiocyanato-butane & 920 & Isothiocyanate & $0.66 \pm 0.15$ & $0.58 \pm 0.15$ & $1.09 \pm 0.19$ & $0.91 \pm 0.09$ & 0.376 & 0.129 & 0.142 \\
\hline
\end{tabular}

\footnotetext{
${ }^{a}$ The table contains those volatile organic compounds (VOCs) that were retained for multivariate analysis. Compounds indicated with a * were identified by comparison of pure standards, the other compounds were tentatively identified according to the same references as cited by van Dam et al. (2010). The numbers in the first column refer to the numbers in the two dimensional bubble plot in Fig. 1

${ }^{\mathrm{b}} \mathrm{RI}=$ retention index on the GC-MS; column RTX5-MS. Peak areas of compounds are means \pm SE

${ }^{\mathrm{c}}$ The $P$-values were determined from a permutation test of the weight vector value which reflects the importance of each compound in the difference between herbivory by one form of herbivory and the control: root herbivory by Delia radicum larvae $=P(\mathrm{D})$; leaf herbivory by Pieris brassicae caterpillars $=P(\mathrm{P})$; dual herbivory by Delia radicum larvae and Pieris brassicae caterpillars $=P(\mathrm{DP})$. Bold face type indicate compounds for which the weight vector value is significant for a specific treatment $(P \leq 0.050)$
} 
Table $2 P$-values $\left(P\left(\mathrm{D}^{*} \mathrm{P}\right)\right)$ from two way analysis of variance on peak areas transformed as $\log (\mathrm{X}+1)$, testing the effect of the interaction between root herbivory by Delia radicum larvae (D) and leaf herbivory by Pieris brassicae caterpillars (P) treatments of Brassica rapa subsp. rapa plants for each selected volatile organic compound

\begin{tabular}{llc}
\hline No. & Compound name & $P(\mathrm{D} * \mathrm{P})$ \\
\hline 1 & $(E, E)-4,8,12-$ Trimethyl-1,3,7,11-tridecatetraene & 0.084 \\
2 & $(3 E)-4,8$-Dimethyl-1,3,7-nonatriene & 0.083 \\
6 & $(E, E)$-alpha-Farnesene & $\mathbf{0 . 0 3 2}$ \\
7 & Methylsalicylate & $\mathbf{0 . 0 4 1}$ \\
9 & Hexylacetate & 0.490 \\
10 & (Z)-3-Hexen-1-ol-acetate & 0.223 \\
14 & 2-Octen-1-ol & $\mathbf{0 . 0 0 5}$ \\
28 & 4-Methyltridecane & $\mathbf{0 . 0 3 7}$
\end{tabular}

The numbers in the first column refer to the numbers in the two dimensional bubble plot in Fig. 1. The table contains those compounds of which the weight vector values refered in Table 1 were significant for at least one form of herbivory. Bold face types indicated significant $P$-values $(P \leq 0.050)$

within the blend of HIPVs elicit a response in the chemoreceptors of the parasitoid (Dicke and Baldwin, 2010).

The volatile blend of plants after leaf herbivory by $P$. brassicae was characterized by higher levels of methylsalicylate. The herbivore-induced emissions of this compound were annulled in the presence of the root herbivore. This volatile is known to be active at very low levels (Gouinguené et al., 2005), attractive for carnivorous arthropods under laboratory conditions (Dicke et al., 1990; Ozawa et al., 2000; De Boer and Dicke, 2004), and for hymenopteran parasitoids in the field (James and Price, 2004; Poelman et al., 2009). On the other hand, methylsalicylate diminished the attraction of the parasitoid Diadegma semiclausum, a specialist of another Brassica specialist herbivore (Plutella xylostella) in a mutant of Arabidopsis (Snoeren et al., 2010). Therefore, the lack of attraction of T. rapae towards $P$. brassicae infested plants compared to root infested plants (Pierre et al., 2011) could be attributed at least partly to a higher level of methylsalicylate and/or to lower levels of 4-methyltridecane and salicylaldehyde.

Dual infested plants exhibited a specific increase of one compound, the green leaf volatile hexylacetate. Specific compounds that were induced by leaf or root herbivory (i. e., 4-methyltridecane and methylsalycilate) did not show changes in emission levels after dual herbivory. Therefore, it appears that simultaneous herbivory significantly reduces or even suppresses the induction of specific compounds that characterize the volatile profile of single infested plants. Only one compound, 2-octen-1-ol, decreased after both single herbivory treatments, but not after dual herbivory.
In addition to specific variations, we also observed communal trends in the volatile profiles among the three forms of herbivory. In particular, the levels of the terpenoids TMTT and (E,E)-alpha-farnesene (marginally induced in dual infested plants) increased after any form of herbivory. Furthermore, the levels of the terpenoid DMNT increased after infestation by the leaf herbivore only, or after dual infestation. These terpenoid compounds are attractive for herbivorous and carnivorous (especially parasitoid) insects associated with brassicaceous plants (e.g., Soler et al., 2007a; van Dam et al., 2010). The present study shows that the specificity of each volatile blend may be due to a mixture of a few specific compounds that increase within the more common odor context of terpenoids and green leaf volatiles. The potential role of such background odors in the use of volatiles by foraging parasitoids has already been reviewed (Hilker and McNeil, 2007). In brassicaceous plants, terpenoids and green leaf volatiles often are abundant within HIPV mixtures, irrespective of the herbivore species that damages the plant. Thus, they themselves may not provide specific cues that allow specialist parasitoids to identify their hosts, which suggests that the specificity of the odor cue then may lie in ratios of the emitted volatiles within the blend (Gols and Harvey, 2009). In this way, the minor quantitative changes in dual infested plants may have contributed to the lack of attractiveness towards $T$. rapae and to the lower levels of parasitism of $D$. radicum in the field (Pierre et al., 2011). The reduced attractiveness of $T$. rapae towards dual infested plants may be adaptive, as both survival and size of T. rapae developing in $D$. radicum on plants infested by $P$. brassicae are reduced (Soler et al., 2007b). The combination of the host $D$. radicum and the non-host $P$. brassicae on the same plant often occurs in the field (Pierre et al., 2011), and it is likely that the parasitoid has learned to detect the characteristic volatile blend of this combination. Similar trends also were found for reverse interactions: root herbivory by $D$. radicum altered the foraging and egg laying behavior of $C$. glomerata, a parasitoid of $P$. brassicae (Soler et al., 2007a). In this case, the volatile blend of dual infested plants also differed only by a few compounds; the emissions of two sulfur compounds were higher, whereas two terpenoids were less prominent in dual infested plants (Soler et al., 2007a). These minor modifications of the complex plant odor blend were hypothesized to be sufficient for the aboveground parasitoids to avoid plants with root herbivores.

The mechanisms involved in the non-additive modification of the odor blends emitted by $D$. radicum and $P$. brassicae infested turnips remain to be elucidated. Crosstalk between signaling hormones, such as jasmonic acid and salicylic acid, involved in responses to different herbivore species may have contributed (van Poecke and Dicke, 2002; Erb et al., 2008; Koornneef and Pieterse, 2008; Zhang et al., 2009). Alternatively, interference 
between HIPV biosynthetic pathway activation by root and shoot herbivory may be involved. A recent study suggested that root induced responses are regulated fundamentally differently from responses triggered by shoot herbivory (van Dam et al., 2010). It was found that the biosynthesis of sesqui- and homoteperne volatiles was induced only by shoot jasmonic acid treatment of $B$. oleraceae plants, whereas monoterpenes were equally elicited by root and shoot jasmonic acid treatment. In the present study, we observed an interaction between root herbivory and leaf herbivory at the level of sesquiterpene $(E, E)$-alpha-farnesene. Therefore, these results support the hypothesis that root and shoot plant responses interfere, leading to variations in the dual infested plant odor mixture. A molecular approach that analyzes the expression of genes coding for markers of signaling or biosynthetic pathways could help to reveal which one is activated or suppressed in response to dual herbivory. Our chemical analysis supports previous behavioral studies in the multitrophic system, turnip- $D$. radicum-P. brassicae- $T$. rapae. Attraction of the parasitoid towards host infested plants was cancelled when these plants were also infested by the leaf feeder $P$. brassicae (Pierre et al., 2011). Analysis of volatile profiles revealed minor quantitative differences between plants infested by a single herbivore species and dual infested plants. However, these subtle changes were found to be highly specific, and thus may be sufficient for T. rapae to discriminate between the different HIPV mixtures. Our data interpretation using OPLS-DA, a novel multivariate analysis approach, has enabled us to identify the minimal, but relevant, changes within a complex HIPV background. This approach provides insight into the information processing of insects that base their behavioral responses upon quantitative differences in HIPV profiles as well as on qualitative contrasts within the context of an odor blend (Hilker and Mcneil, 2007; van Dam and Poppy, 2008). However, additional bioassays coupled with chemical analysis are required to conclude with certainty which compounds or combination of compounds within the plant volatile blend are behaviorally active towards the parasitoid T. rapae.

Acknowledgements The authors thank two anonymous reviewers and Dr. D. Webb for constructive comments on an earlier version of this manuscript. We thank C.E. Raaijmakers, R. Wagenaar, and G. Disveld for assistance in plant maintenance and volatile collection set up, R. Soler and J. Harvey at NIOO-KNAW (Netherlands) for constructive comments during the experiment, L. Koopman at the laboratory of Entomology, Wageningen University for supplying $P$. brassicae cultures, V. Chaminade, C. Josso, and S. Dourlot at Rennes University (France) for supplying D. radicum cultures, M. Hervé at Rennes University (France) for precious advice in statistics. P.S. Pierre thanks the European Science Foundation (ESF) for funding her work with a VOCBAS exchange grant, no 2076. This work also was supported by a PhD grant to P.S. Pierre from the Region Bretagne.

\section{References}

Ali, J. G., Alborn, H. T., and Stelinski, L. L. 2010. Subterranean herbivore-induced volatiles released by Citrus roots upon feeding by Diaprepes abbreviatus recruit entomopathogenic nematodes. J. Chem. Ecol. 36:361-368.

Aratchige, N. S., Lesna, I., and Sabelis, M. W. 2004. Belowground plant parts emit herbivore-induced volatiles: olfactory responses of a predatory mite to tulip bulbs infested by rust mites. Exp. Appl. Acarol. 33:21-30.

Arimura, G. I., Matsui, K., and TAKabayashi, J. 2009. Chemical and molecular ecology of herbivore-induced plant volatiles: proximate factors and their ultimate functions. Plant Cell Physiol. 50:911-923.

BEZEMER, T. M., and van DAM, N. M. 2005. Above- belowground interactions via induced plant defenses. Trends Ecol. Evol. 20:617-624.

Bylesjö, M., Rantalainen, M., Cloarec, O., Nicholson, J. K., Holmes, E., and TRYGG, J. 2006. OPLS discriminant analysis: combining the strengths of PLS-DA and SIMCA classification. $J$. Chemom. 20:341-351.

vAN DAM, N. M., and POPPY, G. M. 2008. Why plant volatile analysis needs bioinformatics - detecting signal from noise in increasingly complex profiles. Plant Biol. 10:29-37.

DE Boer, J. G., and DicKe, M. 2004. The role of methylsalicylate in prey searching behavior of the predatory mite Phytoseiulus persimilis. J. Chem. Ecol. 30:255-271.

De Boer, J. G., Posthumus, M. A., and Dicke, M. 2004. Identification of volatiles that are used in discrimination between plants infested with prey or non prey herbivores by a predatory mite. J. Chem. Ecol. 30:2215-2221.

De Moraes, C. M., Lewis, W. J., Paré, P.W., and Tumlinson, J. H. 1998. Herbivore infested plants selectively attract parasitoids. Nature 393:570-573.

DiCKE, M., and BALDWIN, I. T. 2010. The evolutionary context for herbivore-induced plant volatiles: beyond the "cry for help". Trends Plant Sci. 15: 167-175.

DiCKE, M., and SABELIS, M. W. 1987. How plants obtain predatory mites as bodyguards. Neth. J. Zool. 38:148-165.

DiCKE, M., and vAN LOON, J. J. A. 2000. Multitrophic effects of herbivore-induced plant volatiles in an evolutionary context. Entomol. Exp. Appl. 97:237-249.

Dicke, M., van BeeK, T. A., Posthumus, M. A., Ben Dom, N., van BoKhoven, H., and De Groot, A. E. 1990. Isolation and identification of volatile kairomone that affects acarine predator prey interactions Involvement of host plant in its production. $J$. Chem. Ecol. 16:381-396.

Dicke, M., van LOON, J. J. A., and SOLER R. 2009. Chemical complexity of volatiles from plants induced by multiple attack. Nat. Chem. Biol. 5:317-324.

Dudareva, N., Pichersky, E., and Gershenzon, J. 2004. Biochemistry of plant volatiles. Plant Physiol. 135:1893-1902.

Erb, M., Ton, J., Degenhardt, J., and Turlings, T. C. J. 2008. Interactions between arthropod-induced aboveground and belowground defenses in plants. Plant Physiol. 146:867-874.

Gaquerel, E., Weinhold, A., and Baldwin, I. T. 2009. Molecular interactions between the specialist herbivore Manduca sexta (Lepidoptera, Sphigidae) and its natural host Nicotiana attenuata. VIII. An unbiased GCxGC-ToFMS analysis of the plant's elicited volatile emissions. Plant Physiol. 149:14081423.

Geervliet, J. B. F., Vet, L. E. M., and Dicke, M. 1994. Volatiles from damaged plants as major cues in long-range host-searching by the specialist parasitoid Cotesia rubecula. Entomol. Exp. Appl. 73:289-297. 
Gols, R., and Harvey, J. A. 2009. Plant-mediated effects in the Brassicaeae on the performance and behaviour of parasitoids. Phytochem. Rev. 8:187-206.

Gouinguené, S., Alborn, H., and Turlings, T. C. 2003. Induction of volatile emissions in maize by different larval instars of Spodoptera littoralis. J. Chem. Ecol. 29:145-162.

Gouinguené, S., Pickett, J. A., Wadhams, L. J., Birkett, M. A., and TuRLingS, T. C. J. 2005. Antennal electrophysiological responses of three parasitic wasps to caterpillar-induced volatiles from maize (Zea mays mays), cotton (Gossypium herbaceum), and cowpea (Vigna unguiculata). J. Chem. Ecol. 31:1023-1038.

Gross, J., and SchmidtBerg, H., 2009. Glands of leaf beetle larvae protective structures against attacking predators and pathogens. pp 172-189 in P. JOLIVET, J. SANTIAGO-BLAY and M. SCHMITT (eds.). Research on Chrysomelidae 2. Koninklijke Brill, Leiden, The Netherlands.

Gruber, M. Y, Xu, N., Grenkow, L., Li, X., OnYilagha, J., Soroka, J. J., Westcott, N. D., and Hegedus, D. D. 2009. Responses of the crucifer flea beetle to Brassica volatiles in an olfactometer. Environ. Entomol. 38:1467-1479.

HEIL, M. 2008. Indirect defense via tritrophic interactions. New Phytol. 178:41-61.

HiLKer, M., and MCNEIL, J. N. 2007. Chemical and behavioral ecology in insect parasitoids: how to behave optimally in a complex odorous environment? pp 92-112 in E. WAJNBERG, C. BERNSTEIN, and J. VAN ALPHEN (eds.). Behavioural Ecology of Insect Parasitoids: From Theoretical Approaches to Field Applications. Blackwell Publishing.

JakobSEn, H. B., FriIs, P., Nielsen, J. K., and Olsena, C. E. 1994. Emission of volatiles from flowers and leaves of Brassica napus in situ. Phytochemistry 37:695-699.

JAMES, D.G., and PRICE, S. T. 2004. Field-testing of methylsalicylate for recruitment and retention of beneficial insects in grapes and hops. J. Chem. Ecol. 30:1613-1628.

Jansen, J.J., Smit, S., Hoefsloot, H. C, and Smilde, A. K. 2010. The photographer and the greenhouse: how to analyse plant metabolomics data. Phytochem. Anal. 21:48-60.

KoornneEF, A., and Pieterse, C. M. J. 2008. Update on cross talk in defense signalling. Plant Physiol. 146:839-844.

MARKUS, K. A., and GU, W. 2010. Bubble plots as a model-free graphical tool for continuous variables advances. pp 65-94 in $\mathrm{H}$. D. VINOD (ed.). Advances in Social Research Using R, Lecture Notes in Statistics 196.

MuMM, R., and HILKER, M. 2005. The significance of background odour for an egg parasitoid to detect plants with host eggs. Chem. Sens. 30:337-343.

NeVeU, N., KACEM, N., and NENON, J. P. 1996. A method for rearing Trybliographa rapae W. on Delia radicum L. OILB/SROP Bull. 19:173-178.

Neveu, N., Grandgirard, J., Nenon, J. P., and Cortesero, A. M. 2002. Systemic release of herbivore-induced plant volatiles by turnips infested by concealed root-feeding larvae Delia radicum L. J. Chem. Ecol. 28:1717-1732.

Ozawa, R., Shimoda, T., Kawaguchi, M., Arimura, G., Horiuchi, J., NishioKA, T., and TAKABAYASHI, J. 2000. Lotus japonicus infested with herbivorous mites emits volatile compounds that attract predatory mites. J. Plant Res. 113:427-433.

Pierre, P. S., Dugravot, S., Ferry, A., Soler, R., van Dam, N. M., and CORTESERO, A-M. 2011. Aboveground herbivory affects indirect defences of brassicaceous plants against the root feeder Delia radicum: laboratory and field evidence. Ecol. Entomol. (in press).
Poelman, E. H., Oduor, A. M. O., Broekgaarden, C., HordiJK, C. A., JANSEN J. J.,van LOON, J. J. A., van DAM, N. M., Vet, L. E. M., and DiCKE, M. 2009. Field parasitism rates of caterpillars on Brassica oleracea plants are reliably predicted by differential attraction of Cotesia parasitoids. Funct. Ecol. 23:951-962.

RANGANATHAN, Y., and BORGES, R. M. 2010. Reducing the babel in plant volatile communication: using the forest to see the trees. Plant Biol. 12:689-830.

Rasmann, S., and TuRlingS, T. C. J. 2007. Simultaneous feeding by aboveground and belowground herbivores attenuates plantmediated attraction of their respective natural enemies. Ecol. Lett. 10:926-936.

Rasmann, S., Kollner, T. G., Degenhardt, J., Hiltpold, I., Toepfer, S., Kuhlmann, U., Gershenzon, J., and Turlings, T. C. J. 2005. Recruitment of entomopathogenic nematodes by insect-damaged maize roots. Nature 434:732-737.

Snoeren T. A., Mumm R., Poelman E. H., Yang Y., Pichersky E., and DICKE M. 2010. The herbivore-induced plant volatile Methylsalicylate negatively affects attraction of the parasitoid Diadegma semiclausum. J. Chem. Ecol. 36:479-489.

Soler, R., Harvey, J. A., Kamp, A. F. D., Vet, L. E. M., van der Putten, W. H., van Dam, N. M., Stuefer, J. F., Gols, R., HordiJK, C. A., and BEZEMER, T. M. 2007a. Root herbivores influence the behaviour of an aboveground parasitoid through changes in plant-volatile signals. Oikos 116:367-376.

Soler, R., Bezemer, T. M., Cortesero, A. M., van der Putten, W. H., Vet, L. E. M., and HaRveY, J. A. 2007b. Impact of foliar herbivory on the development of a root-feeding insect and its parasitoid. Oecologia 152:257-264.

TAKABAYASHI, J., and DiCKE, M. 1996. Plant-carnivore mutualism through herbivore-induced carnivore attractants. Trends Plant Sci. $1: 109-113$.

Takabayashi, J., Takahashi, S., Dicke, M., and Posthumus, M. A. 1995. Developmental stage of herbivore Pseudaletia separata affects production of herbivore-induced synomone by corn plants. J. Chem. Ecol. 21:273-287.

Takabayashi, J., Sabelis, M. W., Janssen, A., Shiojiri, K., and vAN WIJK, M. 2006. Can plants betray the presence of multiple herbivore species to predators and parasitoids? The role of learning in phytochemical information networks. Ecol. Res. 21:3-8.

Turlings, T. C. J., Loughrin, J. H., Mccall, P. J., Rose, U. S., LEWIS, W. J., and TUMLINSON J. H. 1995. How caterpillardamaged plants protect themselves by attracting parasitic wasps. Proc. Natl. Acad. Sci. USA. 92:4169-4174.

van Dam, N. M., Tytgat, T. O. G., and KirkegaArd, J. A. 2009. Root and shoot glucosinolates: a comparison of their diversity, function and interactions in natural and managed ecosystems. Phytochem. Rev. 8:171-186.

vaN DAM, N. M., QIU, B. L., HordiJK, C.A., Vet, L. E. M., and JANSEN, J. J. 2010. Identification of biologically relevant compounds in aboveground and belowground induced volatile blends. J. Chem. Ecol. 36:1006-1016.

van Poecke, R. M. P., and Dicke, M. 2002. Induced parasitoid attraction by Arabidopsis thaliana: involvement of the octadecanoid and the salicylic acid pathway. J. Exp. Bot. 53:1793-1799.

VET, L. E. M., WÄCKERS, F. L., and DiCKE, M. 1991. How to hunt for hiding hosts: the reliability-detectability problem in foraging parasitoids. Neth. J. Zool. 41:202-213.

Zhang, P. J., ZHENG, S-J., van LoON, J. J. A., Boland, W., DAVID, A., Mumm, R., and Dicke, M. 2009. Whiteflies interfere with indirect plant defense against spider mites in Lima bean. Proc. Natl. Acad. Sci. USA. 106:21202-21207. 